\title{
Motoneuron Differentiation of Induced Pluripotent Stem Cells from SOD1G93A Mice
}

\author{
Xiao-Li Yao ${ }^{19}$, Cheng-Hui Ye ${ }^{1,29}$, Qiang Liu' ${ }^{1}$, Jian-bo Wan ${ }^{3}$, Jun Zhen ${ }^{4}$, Andy Peng Xiang ${ }^{5}$, Wei-Qiang Li ${ }^{5}$, \\ Yitao Wang $^{3}$, Huangxing $\mathrm{Su}^{3 *}$, Xi-Lin $\mathrm{Lu}^{1 *}$
}

1 Department of Neurology, The First Affiliated Hospital, Sun Yat-sen University, Guangzhou, Guangdong, People's Republic of China, 2 Department of Geriatrics, The First Affiliated Hospital, Sun Yat-sen University, Guangzhou, Guangdong, People's Republic of China, 3 State Key Laboratory of Quality Research in Chinese Medicine, Institute of Chinese Medical Sciences, University of Macau, Macao, People's Republic of China, 4 Department of Rehabilitation Medicine, The Fifth Affiliated Hospital, Sun Yat-sen University, Zhuhai, Guangdong, People's Republic of China, $\mathbf{5}$ Center for Stem Cell Biology and Tissue Engineering, Key Laboratory for Stem Cells and Tissue Engineering, Ministry of Education, Sun Yat-Sen University, Guangzhou, Guangdong, People's Republic of China

\begin{abstract}
Amyotrophic lateral sclerosis (ALS) is a neurodegenerative disorder mainly affecting motor neurons. Mutations in superoxide dismutase-1 (SOD-1) account for about $20 \%$ of familial ALS patients. A robust supply of motoneurons carrying the mutated gene would help understand the causes of motoneuron death and develop new therapeutics for the disease. Here, we established induced pluripotent stem (iPS) cell lines from SOD1G93A mice and compared their potency in motoneuron generation with normal iPS cells and mouse embryonic stem cells (E14). Our results showed that iPS cells derived from SOD1G93A mice possessed the similar potency in neuronal differentiation to normal iPS cells and E14 cells and can be efficiently driven to motoneuron-like phenotype. These cells exhibited typical neuronal morphology, expressed key motoneuron markers, including ChAT and HB9, and generated repetitive trains of action potentials. Furthermore, these neurons highly expressed human SOD-1 and exhibited shorter neurites compared to controls. The present study provides evidence that ALS-iPS cells can be used as disease models in high-throughput screening and mechanistic studies due to their ability to efficiently differentiate into specific neuronal subtypes.
\end{abstract}

Citation: Yao X-L, Ye C-H, Liu Q, Wan J-b, Zhen J, et al. (2013) Motoneuron Differentiation of Induced Pluripotent Stem Cells from SOD1G93A Mice. PLoS ONE 8(5): e64720. doi:10.1371/journal.pone.0064720

Editor: Laurent Coen, Muséum National d'Histoire Naturelle, France

Received November 14, 2012; Accepted April 17, 2013; Published May 28, 2013

Copyright: (C) 2013 Yao et al. This is an open-access article distributed under the terms of the Creative Commons Attribution License, which permits unrestricted use, distribution, and reproduction in any medium, provided the original author and source are credited.

Funding: This study was supported by grants from the National Key Clinical Department, National Key Discipline and Guangdong Key Laboratory For Diagnosis And Treatment of Major Neurological Diseases. This study was supported by National Natural Science Foundation of China (81041056) and Science and Technology Planning Program of Guangdong Province (2010B050700024, 2012B031800107). This study was also supported by multi-year research grant university of Macau, MYRG122 (Y1-L3)-ICMS12-SHX. The funders had no role in study design, data collection and analysis, decision to publish, or preparation of the manuscript.

Competing Interests: The authors have declared that no competing interests exist.

*E-mail: goodxilin@163.com. (XLL); Huanxingsu@umac.mo (HS)

9 These authors contributed equally to this work.

\section{Introduction}

Amyotrophic lateral sclerosis (ALS) is an adult-onset neurodegenerative disease characterized by the selective loss of motoneurons in the cerebral cortex, brainstem, and spinal cord, leading to atrophy of limb, axial, and respiratory muscles [1]. Mutations in superoxide dismutase-1 (SOD-1) account for about 20\% of familial ALS patients [2,3]. SOD1G93A mice is a widely accepted model for the ALS research, which express mutant G93A of human SOD-1 and develop clinical symptoms similar to those seen in ALS patients [4]. Motoneurons from SODlG93A mice could give some information to study the mechanism of ALS [5,6]. A robust supply of motoneurons carrying the genes responsible for this condition would help understand the causes of motoneuron death in ALS and develop new therapeutics for the disease.

Recently, somatic cells can be reprogrammed to a pluripotent state through viral transduction of four transcription factors Oct4, Sox2, c-Myc, and Klf4 [7-9]. The induced pluripotent stem (iPS) cells were indistinguishable from ES cells in proliferative and developmental potential, and they can differentiate into derivatives of all germ layers. Several protocols have been developed to induce iPS cells to efficiently differentiate into neurons [10-14]. However, it remains unknown whether iPS cells with genetic deficiency possess neuronal differentiation potential similar to normal cells lines.

In this study, we compared the neuronal differentiation potential between iPS cells derived from SOD1G93A mice and iPS cells derived from normal C57BL/6 mice and investigated whether SOD1 mutations could influence the neuronal differentiation, especially motoneuron generation from iPS cells. Results of the present study would provide evidence on the possibility of the efficient generation of motoneurons from iPS cells with SOD mutations.

\section{Results}

Generation and characterization of iPS cells from tail-tip fibroblasts

Totally 6 iPS cell lines were generated by retroviral expression of mouse Oct4, Sox2, c-Myc, and Klf4 from B6SJL-TgN TTFs and C57BL/6 TTFs for characterization and comparison, in which 3 iPS cell lines were derived from 3 transgenic B6SJL-TgN 
mice (ALS-iPS) and 3 iPS cell line were derived from 3 C57BL/6 mice (C57-iPS) (Figs. 1A and 1C). To confirm that these iPS cells exhibit ES-like properties, we examined some ES cell markers that included alkaline phosphatase (AP) activity and ES cell-specific transcription factors Oct4 and SSEA-1. Results shown in Figs. 1B and $1 \mathrm{D}$ demonstrated that the iPS clones exhibited high AP activity. The selected iPS clones were also shown to be positive for Oct4 and SSEA-1 (Figs. 2A and 2B). To assess the gene expression pattern of the iPS clones, we isolated RNA from iPS cells and the result indicated that the endogenous Oct4, Sox2, c-Myc, Klf4, and Nanog were expressed which confirmed activation of these loci. Results shown in Fig. 2C demonstrated that the transgenes of selected clones from both ALS-iPS-1 and C57-iPS-12 cells were silenced. Importantly, all analyzed iPS clones induced expression from the endogenous Oct4, Sox2, and Nanog loci, and none of these genes were expressed in the original TTF fibroblasts, further supporting of successful reprogramming. Karyotype analyses demonstrated that all analyzed ALS-iPS-1 clones (Fig. 2G) and C57-iPS-12 clones (data not shown) exhibited a normal karyotype.

To confirm the pluripotency of the iPS cells, we injected iPS cells intramuscularly into nude mice. Teratomas formed 4-6 weeks after injection. HE staining of tumor sections from teratomas dissected 5 weeks after injection demonstrated the presence of cell types of all the 3 germ layers (Figs. 2D-F), including gland tissues (endoderm, Fig. 2D), cartilage tissue (mesoderm, Fig. 2E), and neural tube (ectoderm, Fig. 2F).

\section{Transduction and expression of T $\alpha 1 \alpha$-tubulin/hrGFP (T $\alpha 1)$ during iPS cell neuronal differentiation}

To facilitate to monitor the neuronal differentiation potential, we transduced iPS cell lines (ALS-iPS-1 and C57-iPS-12) with lentivector containing $\mathrm{T} \alpha 1 \alpha$-tubulin promoter. E14 cells were used as control. After antibiotic selection, tansduced undifferentiated mouse iPS cells and E14 cells were obtained.
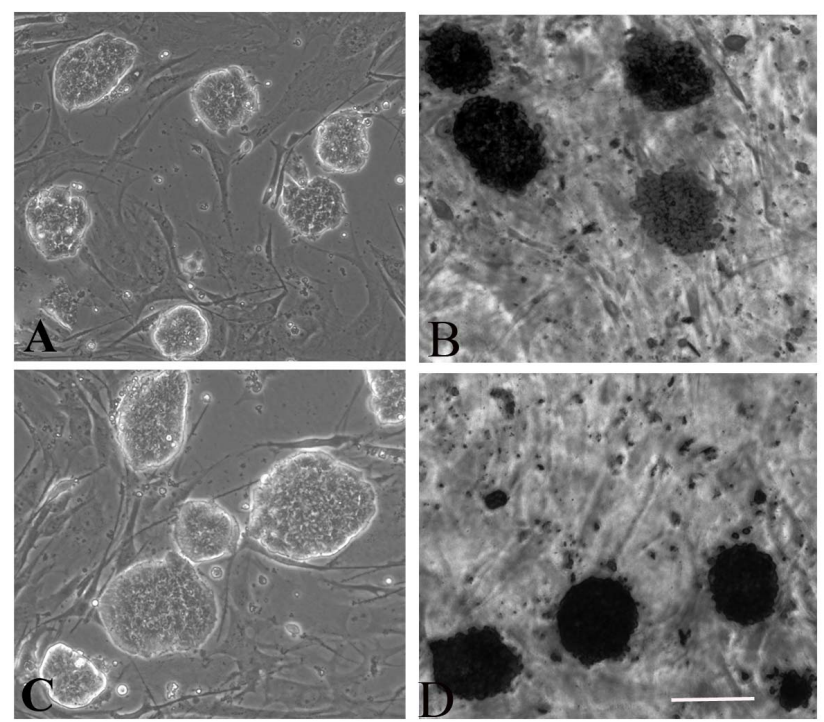

Figure 1. Establishment of mouse iPS cell lines from SOD1G93A mice and C57BL/6 mice. (A) Phase contrast image shows that iPS cells from SOD1G93A mice (ALS-iPS-1) grew as colonies on mitomycin-treated MEF feeder cells. (B) These clones exhibited high AP activity. (C) Phase contrast image shows that iPS cells from C57BL/6 mice grew as colonies on mitomycin-treated MEF feeder cells. (D) These clones exhibited high AP activity. Scale bar: $500 \mu \mathrm{m}$. doi:10.1371/journal.pone.0064720.g001
Under the control of the T $\alpha 1 \alpha$-tubulin promoter, GFP expression appeared at day 2 of the EBs (Fig. 3). After 4 days treatment with RA, the expression of GFP increased over time (Fig. 3). To identify and separate neurons from differentiated E14 and iPS cells, we used a strategy of GFP-based FACS. FACS analysis indicated that $12.4 \pm 2.6 \%$ of ALS-iPS-1 differentiated cells was GFP positive, $13.2 \pm 2.4 \%$ of C57-iPS-12 differentiated cells was GFP positive, while $13.1 \pm 1.8 \%$ of E14 differentiated cells was GFP positive cells, showing no statistical difference in the number of GFP-positive among the three cell lines $(\mathrm{P}>0.05)$ (Fig. 3J). After fluorescent-activated cell sorting, the GFP positive cells were enriched to high purity. The sorted GFP-expressing cells were stained positively for $\beta$ III-tubulin. Quantitative analysis of these immunocytostaining experiments demonstrated that 95.2 $\pm 4.7 \%(403 / 423, \mathrm{n}=6)$ of the sorted cells from ALS-iPS-1 cells were stained positively for $\beta$-III-tubulin, 94.4 $\pm 6.5 \%$ (425/ $450, \mathrm{n}=6$ ) of the sorted cells from C57-iPS-12 cells were stained positively for $\beta$-III-tubulin, while $95.0 \pm 5.6 \% \quad(419 / 441, n=6)$ from E14 cells were stained positively for $\beta$-III-tubulin, suggesting that nearly all GFP-positive cells were differentiated neurons (Fig. 4).

\section{Motoneuron differentiation and characterization of iPS cell lines}

Incubation with RA and $\mathrm{SHH}$ led to efficient motoneuron generation from iPS and E14 cell lines. Approximately $83.5 \pm 7.9 \% \quad \beta$-III-tubulin-positive neurons co-expressed ChAT in ALS-iPS-1 cell culture after 5 days treatment with RA and SHH (Fig. 5A4). When stained with $\mathrm{Hb9}$ (a specific postmitotic motoneuron marker), $24.3 \pm 2.7 \%$ Tuj1-positive neurons were found to be Hb9-positive (Fig. 5A3). C57-iPS-12 cells showed similar potency in motoneruon generation. $85.2 \pm 6.8 \% \quad \beta$-IIItubulin-positive neurons co-expressed ChAT (Fig. 5B4) and $26.1 \pm 3.4 \% \quad \beta$-III-tubulin-positive neurons co-expressed $\mathrm{Hb} 9$ (Fig. 5B3) in C57-iPS-12 cell culture after 5 days treatment with $\mathrm{RA}$ and SHH. There is no difference in the percentage of both ChAT-positive neurons and Hb9-positive neurons between ALSiPS-1 and C57-iPS-12 groups (ChAT: $83.5 \pm 7.9 \%$ vs $85.2 \pm 6.8 \%$, $\mathrm{P}>0.05$; Hb9: $24.3 \pm 2.7 \%$ vs $26.1 \pm 3.4 \%, \mathrm{P}>0.05)$, suggesting that SOD1 mutation did not reduce the potency of motoneuron differentiation compared to the normal iPS cell line. Efficient motoneuron generation from E14 cells was also observed. Five days treatment with $\mathrm{RA}$ and $\mathrm{SHH}$ led to $88.6 \pm 9.4 \% \quad \beta$-IIItubulin-positive neurons co-expressing ChAT and $27.2 \pm 3.1 \% \beta$ III-tubulin-positive neurons co-expressing Hb9 (Figs. 5C and D). The three cell lines did no differ in the potency of motoneuron generation as revealed by the percentage of ChAT-positive neurons and Hb9-positive neurons (Figs. 5C and D). Standard whole-cell patch clamp, current-clamp techniques were then used to study the electrical properties of these motor neurons (Fig. 5E). Both ALS-iPS and C57-iPS cells-derived motoneurons generated repetitive trains of action potentials, suggesting that they were functional (Fig. 5F). However, ALS-iPS cells-derived motoneurons exhibited shorter neuritis $(50.5 \pm 7.9 \mu \mathrm{m}$ compared to 95.8 $\pm 12.5 \mu \mathrm{m}$ for C57-iPS cells-derived motoneurons, $\mathrm{P}<0.001$, student t-test) (Figs. 6A and B). Quantitative real time RT-PCR showed that these ALS-iPS cells-derived motoneurons highly expressed human SOD-1, while C57-iPS cells-derived did not express any (Fig. 6C).

\section{Discussion}

Using iPSGs technology, researchers can achieve ES-like cells without the ethical dilemma. The derivation of iPS cells is of such 

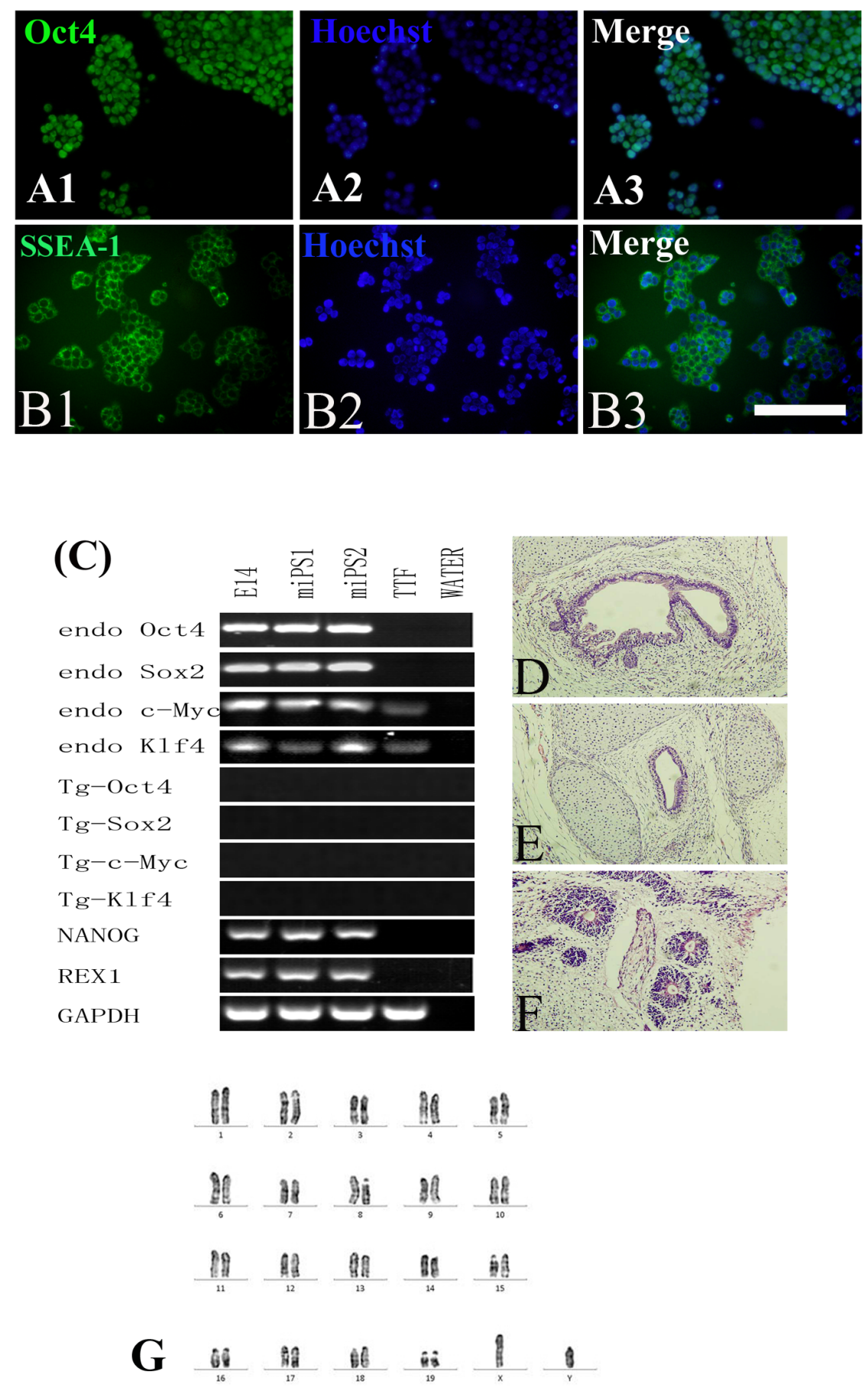

Figure 2. Immunostaining shows that the established iPS cell line (ALS-iPS-1) was positive for Oct4 (A) and SSEA-1 (B). (C) The expression patterns of pluripotent genes in iPS clones, E14 cells, and fibroblasts. The results revealed that all analyzed iPS clones induced expression from the endogenous Oct4, Sox2, and Nanog loci, and none of these genes were expressed in the original TTF fibroblasts. (D-F) Teratoma derived from ALS-iPS-1 cells contained cells belonging to all three germ layers, including endoderm-derived glandular (D), mesoderm-derived cartilage tissue (E), and ectodermderived neural tubes (F). Karyotype analyses demonstrated that ALS-iPS-1 clones showed a normal karyotype (G). Scale bar: $100 \mu \mathrm{m}$ in A and B; $250 \mu \mathrm{m}$ in $\mathrm{D}, \mathrm{E}$, and $\mathrm{F}$.

doi:10.1371/journal.pone.0064720.g002

great importance because of the ease and reproducibility of generating them. Direct reprogramming provides, for the first time, a realistic way of generating sufficient numbers of patientspecific pluripotent stem cells. Such cells could be used for regenerative and therapeutic purposes, as demonstrated in mouse models of, for example, sickle cell anemia and Parkinson's disease, respectively $[15,16]$. In this study, we generated iPS cells from TTFs of SOD1G93A mice by retroviral constructs encoding Oct4, Sox2, c-Myc and Klf4 and demonstrated that they possessed the similar potency in neuronal differentiation to normal iPS cells and mouse ESCs (E14), providing evidence that they can be used as 

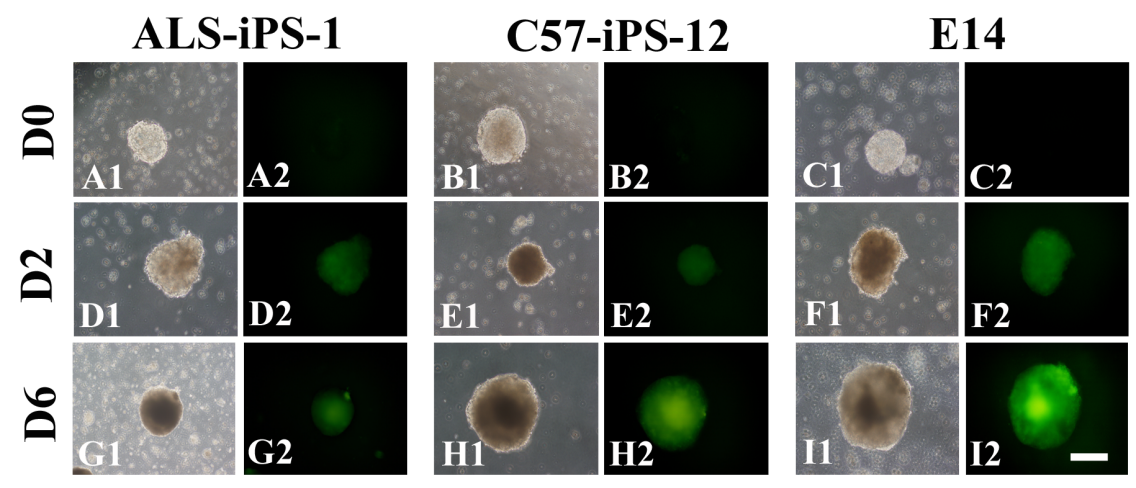

(J)

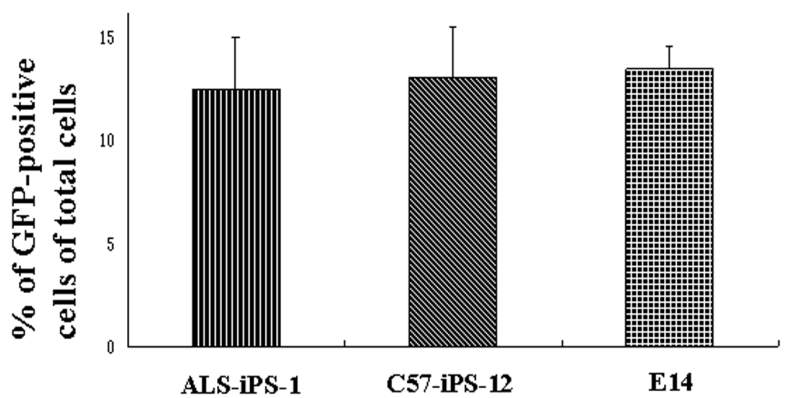

Figure 3. Live monitoring of T $\alpha 1 \alpha$-tubulin promoter driven GFP in the neural differentiation process. At day 0, undifferentiated ALS-iPS1 (A), C57-iPS-12 (B) and E14 (C) did not express GFP. GFP expression appeared at day 2 of the EBs of ALS-iPS-1 (D), C57-iPS-12 (E) and E14 (F). The expression of GFP increased over time (G-I). (J) FACS analysis indicated that $12.4 \pm 2.6 \%$ of ALS-iPS-1 differentiated cells was GFP positive, $13.2 \pm 2.4 \%$ of C57-iPS-12 differentiated cells was GFP positive, while $13.1 \pm 1.8 \%$ of E14 differentiated cells was GFP positive cells, showing no statistical difference in the number of GFP-positive among the three cell lines ( $P>0.05)$. Scale bar: $75 \mu \mathrm{m}$.

doi:10.1371/journal.pone.0064720.g003
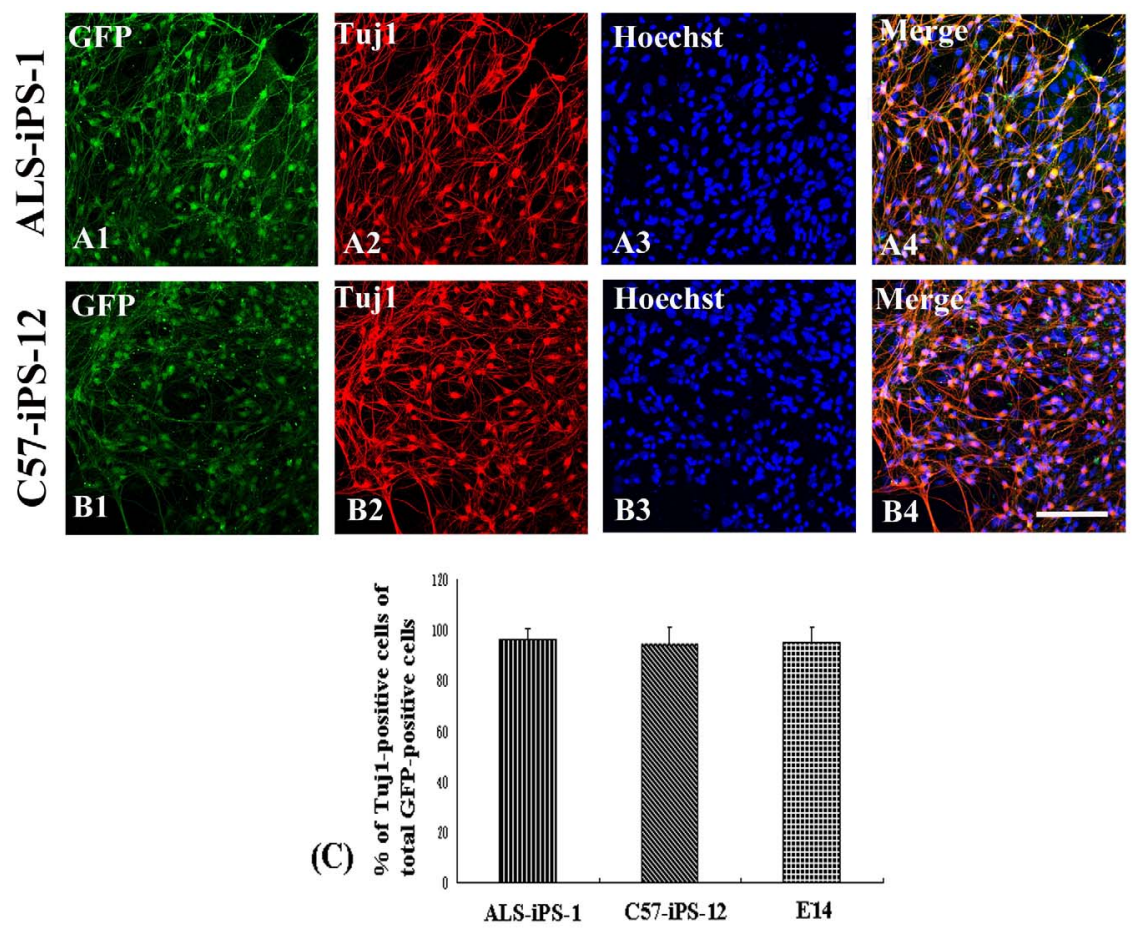

Figure 4. Immunocytostaining of differentiated neurons. FACS sorted GFP-positive cells were cultured on the poly-L-ornithin/laminin-coated plates for $24 \mathrm{hr}$, and then analyzed by immunofluorescence. The GFP-expressing cells were well overlapped with $\beta$-III-tubulin (Tuj1) immunolabeling. (A) ALS-iPS-1 cells. (B) C57-iPS-12 cells. (C). Quantification data demonstrated that $95.2 \pm 4.7 \%$ of the sorted cells from ALS-iPS- 1 cells were stained positively for $\beta$-III-tubulin, $94.4 \pm 6.5 \%$ of the sorted cells from C57-iPS-12 cells were stained positively for $\beta$-III-tubulin, while $95.0 \pm 5.6 \%$ from E14 cells were positive for $\beta$-III-tubulin, suggesting that nearly all GFP-positive cells were differentiated neurons. Scale bar: $80 \mu \mathrm{m}$.

doi:10.1371/journal.pone.0064720.g004 

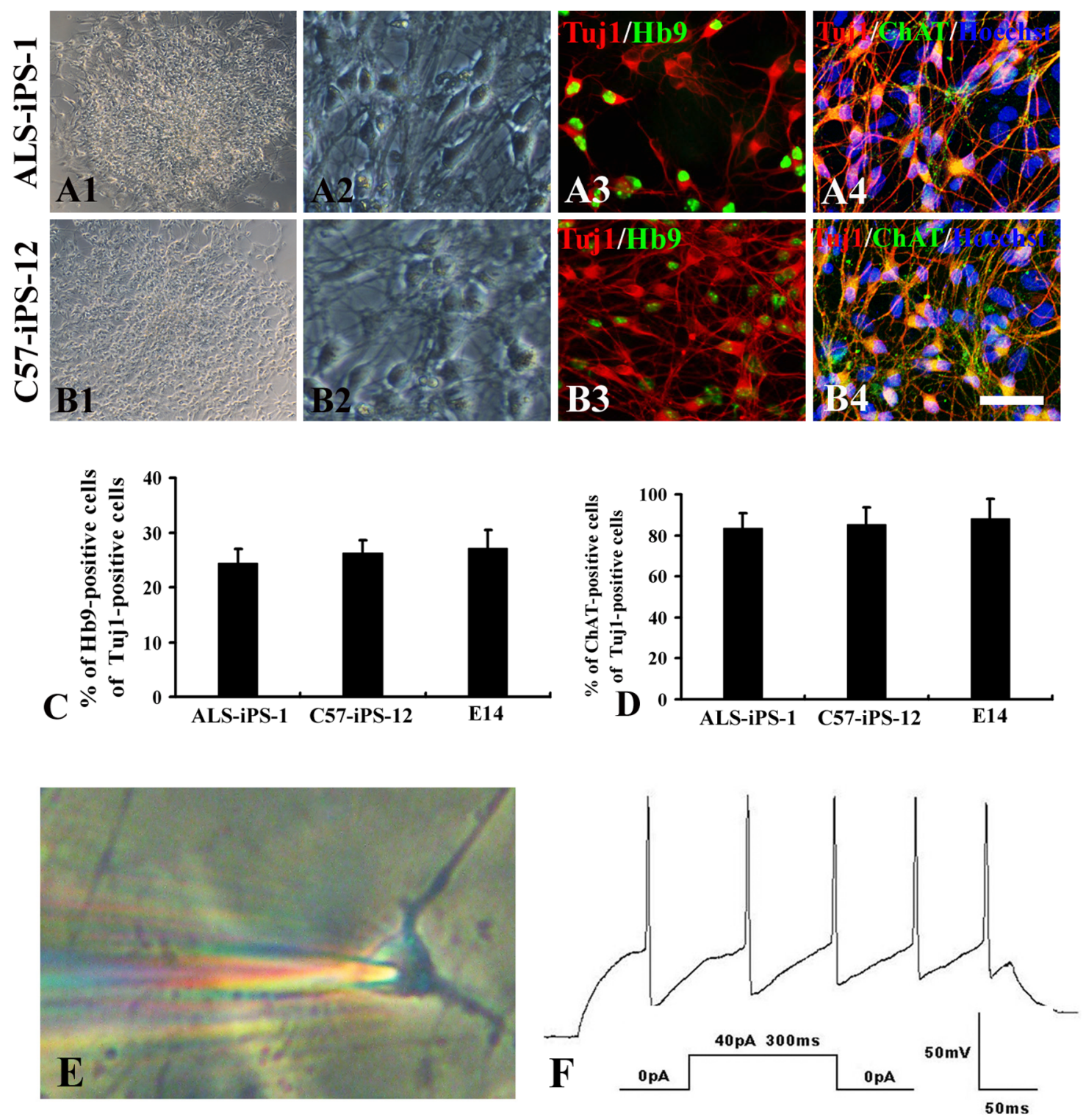

Figure 5. Motoneuron differentiation and characterization. (A). Motoneuron differentiation of ALS-iPS-1. (B). Motoneuron differentiation of C57-iPS-12. (A1 and B1) Phase contrast image shows neuronal differentiation of after 5 days treatment with RA and SHH. (A2 and B2) Higher magnification shows typical neuronal morphology. (A3 and B3) Confocal imaging shows that HB9 was expressed on differentiation to TuJ1-positive neurons. (A4 and B4) Confocal imaging shows that the majority of mouse iPSC-derived Tuj-1-positive neurons co-expressed ChAT. (C). Quantification data show that there is no difference in the percentage of Hb9-positive neurons across the iPS cell lines and ES cell line (P>0.05). (D) Quantification data show that there is no difference in the percentage of ChAT-positive neurons across the iPS cell lines and ES cell line (P>0.05). (E). A phase contrast image showing that a whole-cell patch-clamp recording electrode attached on an iPSC-derived motoneuron-like cell under an IR/DIC microscopy. (F). Single current injection (300 ms duration, $40 \mathrm{pA}$ ) showing these motoneurons generated repetitive trains of action potentials. Scale bar: $200 \mu \mathrm{m}$ in $\mathrm{A} 1$ and $\mathrm{B} 1 ; 80 \mu \mathrm{m}$ in $\mathrm{A} 2-\mathrm{A} 4 ; \mathrm{B} 2-\mathrm{B} 3$.

doi:10.1371/journal.pone.0064720.g005

disease models in high-throughput screening and mechanistic studies.

We used a neuron-specific promoter to monitor the neural differentiation processes of iPS cells. After antibiotic selection, mouse iPS cells and ESGs expressing the T $\alpha 1$ : hGFP with a high degree of purity and stability could be obtained. Our data have shown that it can be used in studies to monitor the differentiation of pluripotent stem cells to early neurons. Modification of derivatives with fluorescent markers allows for the cell separation under a fluorescence-equipped dissecting microscope or by fluorescence activated cell sorting. More than 95\% GFP-positive cells were found to be Tuj1-positive neurons across iPS cell lines and one ES cell line, confirming the validity of the T $\boldsymbol{\alpha} 1 \boldsymbol{\alpha}$-tubulin promoter-based neuronal selection strategy.

The ability to generate iPS cells and efficiently differentiate them into specific neuronal subtypes will provide powerful new tools to study complex neurogenetic disorders. ALS-iPS cellderived motoneurons are an ideal model to study the disease, because they contain the same nuclear genome as of patients, which helps understand the cellular physiology of the disease and development of drug treatment. Using an established protocol, ALS-iPS cells could be driven to motoneuron-like phenotypes 

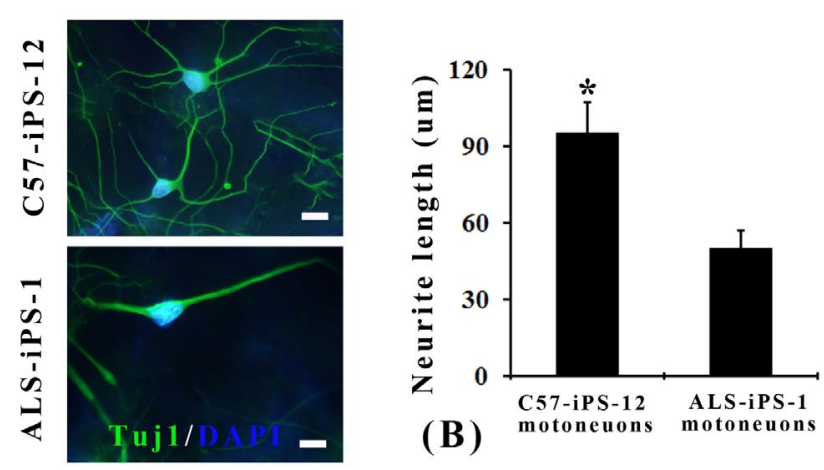

(A)

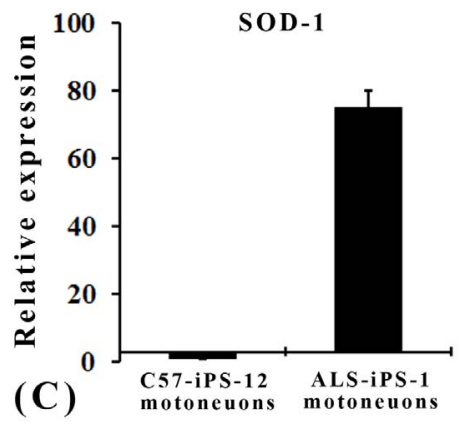

Figure 6. Phenotypes of ALS-iPS-derived motoneurons. (A). Micrographs showing that ALS-iPS cells-derived motoneurons exhibited shorter neuritis than C57-iPS cells-derived motoneurons. (B). Quantification data show that the length of neurites of ALS-iPS-derived motoneurons is $50.5 \pm 7.9 \mu \mathrm{m}$, which is significantly shorter than $95.8 \pm 12.5 \mu \mathrm{m}$ for $\mathrm{C} 57-\mathrm{iPS}$ cells-derived motoneurons $(\mathrm{P}<0.001$, student t-test). (C). Quantitative real time RT-PCR showed that these ALS-iPS cells-derived motoneurons highly expressed human SOD-1, while C57iPS cells-derived did not express any. Scale bar: $15 \mu \mathrm{m}$. doi:10.1371/journal.pone.0064720.g006

efficiently. Our results were supported by the previous findings using SOD1G93A mES cells that the presence of G93A hSODl mutation does not affect early neuron differentiation [17]. These cells expressed key motoneuron markers, including ChAT and HB9, indicating the usefulness of these cells in drug screens and basic research. A key limitation of the study is that no phenotypic changes that may correspond to the disease process were described and future work is also needed to functionally characterize these derived cells for basic neuronal properties.

\section{Conclusion}

Here, we reported a successful derivation of mouse iPS cells from SODlG93A mice and these iPS cells can be induced to differentiation to motorneurons with efficiency similar to that of mouse ES cells and wild type mouse iPS cells. Our preliminary study provides a new ALS disease models for high-throughput screening and mechanistic studies.

\section{Materials and Methods}

\section{Cells culture}

All experimental procedures were approved by the Institutional Animal Ethical Committee of Sun Yat-sen University and were conducted according to the Guide for the Care and Use of Laboratory Animal of the National Institute of Health (Publication No. 80-23, revised 1996).
Fibroblasts were prepared from tail tips of transgenic male mice B6SJL-TgN (SOD1G93A) 1 Gur (G93A; Jackson Laboratories) and adult male C57BL/6 mice as reported [7]. Tail tip fibroblasts (TTFs) were maintained in DMEM containing 10\%FBS and expanded in 1:5 ratio. Murine cell line E14 is purchased from ATCG, American Type Culture Collection (Rockville, MD, USA). iPS cells were cultured in DMEM medium supplemented with 15\% FBS, $2 \mathrm{mM}$ glutamine, $1 \%$ non-essential amino acids, 50 units $/ 50 \mathrm{mg} / \mathrm{ml}$ penicillin-streptomycin and $0.1 \mathrm{mM}$ b-mercaptoethanol (all Invitrogen), and $10 \mathrm{ng} / \mathrm{ml} \mathrm{LIF} \mathrm{(Chemicon,} \mathrm{Teme-}$ cula, California, USA) on gamma irradiation-treated mouse embryonic fibroblast (MEF) feeders at $37^{\circ} \mathrm{C}$ with $5 \% \mathrm{CO} 2$ in air.

\section{Retroviral infection and iPS cell induction}

The protocol for mouse iPS was approved by the Institutional Animal Ethical Committee of Sun Yat-sen University.

Retroviral infection was performed as described by others $[7,8]$ with minor modifications. Plat-E cells are purchased from Cell Biolabs (San Diego, CA 92126 USA). Plat-E cells were seeded at $1 \times 10^{6}$ cells per well of a 6 -well plate. Next day, the cells were transfected with pMXs vectors carrying Oct4, Sox2, c-Myc and Klf4 cDNAs using Lipofectamine 2000 (Invitrogen) according to the manufacturer's instructions. After $12 \mathrm{hr}$, the medium was replaced with fresh medium. Virus-containing supernatants were collected from each plate at $72 \mathrm{hr}$ post-transfection, filtered through a $0.45 \mu \mathrm{m}$ filter before transduction. Equal volumes of the supernatants were mixed and supplemented with $4 \mu \mathrm{g} / \mathrm{ml}$ polybrene. TTFs were seeded at a density of $5 \times 10^{4}$ cells per 6 -well plate and incubated in the virus/polybrene-containing supernatants for $24 \mathrm{hr}$. Four days after infection, the cells were further subcultured on irradiated MEFs in ES medium containing LIF. About two weeks after infection, the colonies were mechanically isolated, and propagated under ES conditions.

\section{Cell line characterization}

Alkaline phosphatase staining, immunofluorescence microscopy, semi-quantitative RT-PCR for transgene integration, karyotyping, and teratoma formation were carried out to characterize iPS cell lines. Direct Alkaline phosphatase (AP) activity was analyzed with the alkaline phosphatase substrate BCIP/NBT (Sigma) according to the manufacturer's guidelines. The following primary antibodies were used: anti-Oct-4 (Santa Cruz Biotechnology, Santa Cruz, CA, USA), SSEAl (Chemicon). Total mRNA was isolated using TRIZOL and $1 \mu \mathrm{g}$ was used to synthesize cDNA using Murine Leukemia Virus reverse transcriptase (Fermentas) and oligo-dT primers (Fermentas) according to the manufacturer's instruction. Then the samples were subjected to amplification with mouse specific primers. $\beta$-actin was used as positive control. The PCR products were analyzed by $1.2 \%$ agarose gel electrophoresis and visualized by ethidium bromide staining. The detailed information of primers is listed in Table 1. To form teratomas, approximately $2 \times 10^{6}$ cells were injected into hind limb muscle of 5 -week-old nude mice. After five weeks, teratomas were dissected and fixed in $4 \%$ paraformaldehyde. Samples were embedded in paraffin and processed with hematoxylin and eosin staining.

\section{Vector construction and lentivirus production}

To generate Lentivirus, 293FT cells (Invitrogen) were transfected at 90\% confluence using Lipofectamine 2000 (Invitrogen). For a $10 \mathrm{~cm}$ plate, $9 \mu \mathrm{g}$ of ViraPower packaging mix and $3 \mu \mathrm{g}$ of pLV/Final-puro-T $\alpha 1 \alpha$-tubulin-hrGFP [18] lentiviral vector were co-transfected into the 293FT cells using $36 \mu \mathrm{l}$ Lipofectamine 
Table 1.

\begin{tabular}{|c|c|}
\hline Gene & Sequence $\left(5^{\prime}\right.$ to $\left.3^{\prime}\right)$ \\
\hline \multirow[t]{2}{*}{ Endo-Oct4 } & СTCTGCTTCAGGAGCTTGG \\
\hline & GAAGGAGAAGCTGGAGCAAA \\
\hline \multirow[t]{2}{*}{ pMX-Oct4 } & CCCCAGGGCCCCATTTGGTACC \\
\hline & TTATCGTCGACCACTGTGCTGCTG \\
\hline \multirow[t]{2}{*}{ Endo-Sox2 } & GGGAAATGGGAGGGGTGCAAAAGG \\
\hline & TTGCGTGAGTGTGGATGGGATTGGG \\
\hline \multirow[t]{2}{*}{ pMX-Sox2 } & GGCACCCCTGGCATGGCTCTTGGCTC \\
\hline & TTATCGTCGACCACTGTGCTGCTG \\
\hline \multirow[t]{2}{*}{ Endo-c-Myc } & GCGTCCTGGGAAGGGAGATCCGGAC \\
\hline & TTGAGGGGCATCGTCGCGGGAGGCG \\
\hline \multirow[t]{2}{*}{ pMX-c-Myc } & CAACAACCGAAAATGCACCAGCCCC \\
\hline & TTGCGTGAGTGTGGATGGGATTGGG \\
\hline \multirow[t]{2}{*}{ Endo-Klf4 } & TGATTGTAGTGCTTTCTGGCTGGGCC \\
\hline & ACGATCGTGGCCCCGGAAAAGGACC \\
\hline \multirow[t]{2}{*}{ pMX-KIf4 } & ACGATCGTGGCCCCGGAAAAGGACC \\
\hline & TTGAGGGGCATCGTCGCGGGAGGCG \\
\hline \multirow[t]{2}{*}{ Nanog } & СCTATGCCTGTGATTTGTGGG \\
\hline & AGGTTGTTTGCCTTGGGAC \\
\hline \multirow[t]{2}{*}{ Rex1 } & CAGATCCTAAACAGCTCGCAGAAT \\
\hline & GCGTACGCAAATTAAAGTCCAGA \\
\hline \multirow[t]{2}{*}{ GAPDH } & GATTTGTGGGCCTGAAGAAA \\
\hline & TGTAGACCATGTAGTTGAGGTCA \\
\hline
\end{tabular}

2000 reagent. Virus supernatant was collected 72 hours later and filtrated through $0.45-\mu \mathrm{m}$ filters.

\section{ES and iPS cell transduction and drug selection}

E14 (American Type Culture Collection, ATCG) and iPS cells were transduced for $12 \mathrm{~h}$ with lentivirus in the presence of $6 \mu \mathrm{g} /$ $\mathrm{ml}$ of polybrene. Puromycin selection $(1-3 \mu \mathrm{g} / \mathrm{mL})$ was started $96 \mathrm{hr}$ post-transduction and lasted for seven days.

\section{Neural differentiation}

Undifferentiated E14 cells and iPS cells were treated with $0.125 \%$ trypsin-EDTA and grown in aggregate cultures for 2 days in DFK10 medium to form embryoid bodies (EBs). DFK10 medium consisted of DMEM/F12 (GIBCO, Invitrogen), knockout serum replacement (10\%), penicillin/streptomycin (1\%), N2 supplement $(2.4 \%)$, glucose $(4.5 \mathrm{mg} / \mathrm{ml})$, L-glutamine $(200 \mathrm{mM})$, heparin $(1 \mathrm{u} / \mu \mathrm{l}$; Sigma), and $\beta$-mercaptoethanol $(0.1 \mathrm{mM})$. After 2 days, EBs were treated with $1 \mu \mathrm{M}$ of all-trans RA (Sigma) for another 4 days. These EBs with 4 days RA treatment were subsequently used for FACS sorting studies.

For motoneuron differentiation, SHH and RA were used as described previously with slight modifications [19,20]. After 2 days culture of dissociated E14 or iPS cells in DFK10 medium to form embryoid bodies (EBs), RA (0.1 $\mu \mathrm{M}$; Sigma) and SHH (200 ng/ $\mathrm{ml}$; R\&D Systems) were added to culture for additional 5 days. To facilitate immunostaining analysis, EBs were plated on laminincoated coverslips in DFK 10 medium at day 5 and subsequently supplemented with $10 \mathrm{ng} / \mathrm{mL}$ BDNF, $10 \mathrm{ng} / \mathrm{mL}$ GDNF, $10 \mathrm{ng}$ / $\mathrm{mL}$ CNTF, and $10 \mathrm{ng} / \mathrm{mL}$ IGF (R\&D Systems) to aid in neuronal survival.

\section{Motoneuron characterization}

Immunocytochemical staining on coverslip cultures was performed to characterize neuronal differentiation. Motoneurons were double-stained with mouse anti-Tuj1 (1:500, Sigma) and goat anti-ChAT (1:400, Millipore) or rabbit anti-Tuj1 (1:200, Sigma) and mouse anti-HB9 (1:50, DSHB). Species-specific fluorescenceconjugated secondary antibodies conjugated to Alexa 568 or 488 (1:400, Molecular Probes) were applied for $2 \mathrm{~h}$ at room temperature. Cell slides were then counterstained with DAPI to stain nuclei, and cover-slipped with antifade mounting media (FluorSave, Calbiochem). The images were taken with a laser confocal microscope (LSM510 META, Carl Zeiss Meditec).

Whole-cell patch-clamp recording was used to study the electrophysiological properties of both ALS-iPS and C57-iPS derived motoneurons in culture. Patch pipettes (resistance 3$5 \mathrm{M} \Omega$ ) were filled with the following (in $\mathrm{mM}$ ): 140 potassium methanesulfonate, 10 HEPES, $5 \mathrm{NaCl}, 1 \mathrm{CaCl}$, 0.2 EGTA, 3 ATP-Na2, 0.4 GTP-Na2, pH 7.3 (adjusted with $\mathrm{KOH}$ ). The external solution contained (in $\mathrm{mM}$ ): $120 \mathrm{NaCl}, 1.2 \mathrm{KH} 2 \mathrm{PO} 4,1.9$ KCl, 26 NaHCO3, 2.2 CaCl2, 1.4 MgSO4, 10 D-glucose, 7.5 HEPES (pH with $\mathrm{NaOH}$ to 7.3). The bath solution was equilibrated with 95\% O2 and 5\% CO2 before use. Resting potentials were maintained at about $-65 \mathrm{mV}$. Whole-cell patchclamp recordings were amplified and filtered using an Axopatch 200B amplifier (Molecular Devices, Sunnyvale, CA). Signals were sampled at $10 \mathrm{kHz}$ using a Digidata $1440 \mathrm{~A}$ analog-to-digital converter and acquired and stored on a computer hard drive using pClamp10 software. All voltage and current-clamp recordings were performed. Data were analyzed using pClamp10 (Clampfit).

Total RNAs of motoneurons derived from ALS-iPS and C57iPS cells were extracted using TRIzol (Invitrogen) respectively. Quantitative real time RT-PCR (qPCR) was performed using a Thermal Cycler DiceTM Real Time System and SYBR Premix EX TaqTM (Takara). The primer for human SOD1 (Forward 5'CAT GAG CCG TAA TCG ATG TGA-3' and Reverse 5' - CGC GAC TAA CAA TCA AAG TGA-3') was used. $\beta$-actin was used for $\mathrm{qPCR}$ normalization, and all items were measured in triplicate.

\section{FACS sorting}

For flow cytometry analysis, differentiated cells on day 6 were trypsinized and filtered through a $40 \mu \mathrm{m}$ nylon mesh to remove cell debris. The cells were resuspended in PBS at a concentration of $1 \times 10^{6}$ cells $/ \mathrm{ml}$. Cell analysis and sorting were performed on a FACS-Aria (BD-Biosciences), through which cell sorting purity of $>97 \%$ was achieved consistently. An aliquot of untransfected E14 cell suspension was used as negative control. The cells were analyzed by light forward and side scatter by a 488-nm laser beam. Sorting procedures were only based on fluorescence intensity and performed with a flow rate of 1500 events/sec. GFP-positive cells were replated on poly-L-ornithin/laminin-coated dishes in N2B27 medium for $24 \mathrm{hr}$ and were then analyzed for $\beta$-III-tubulin expression.

\section{Statistical analysis}

Five wells per experiment were imaged for quantification. Results are the average \pm SEM of data from a minimum of three experiments unless stated otherwise. Around 200 cells were counted for each marker. Statistical analysis was performed using one way ANOVA. In case of small numbers in the contingency table, a two-tailed Fisher's-exact test was used. 


\section{Author Contributions}

Conceived and designed the experiments: XLY HS XLL WQL. Performed the experiments: XLY CHY XLL JW QL JZ. Analyzed the data: XLY

\section{References}

1. Mitchell JD, Borasio GD (2007) Amyotrophic lateral sclerosis. Lancet 369: 2031-2041.

2. Rosen DR, Siddique T, Patterson D, Figlewicz D, Sapp P, et al. (1993) Mutations in $\mathrm{Cu} / \mathrm{Zn}$ superoxide dismutase gene are associated with familial amyotrophic lateral sclerosis. Nature 362: 59-62.

3. Orrell RW (2007) Understanding the causes of amyotrophic lateral sclerosis. N Engl J Med 357: 822-823.

4. Gurney ME, Pu H, Chiu AY, Dal Canto MC, Polchow CY, et al. (1994) Motor neuron degeneration in mice that express a human $\mathrm{Cu}, \mathrm{Zn}$ superoxide dismutase mutation. Science 264: 1772-1775.

5. Boillée S, Yamanaka K, Lobsiger CS, Copeland N, Jenkins NA, et al. (2006) Onset and progression in inherited ALS determined by motor neurons and microglia. Science 312: 1389-1392.

6. Yamanaka K, Chun SJ, Boillee S, Fujimori-Tonou N, Yamashita H, et al. (2008) Astrocytes as determinants of disease progression in inherited amyotrophic lateral sclerosis. Nat Neurosci 11: 251-253.

7. Takahashi K, Yamanaka S (2006) Induction of pluripotent stem cells from mouse embryonic and adult fibroblast cultures by defined factors. Cell 126: 663676.

8. Takahashi K, Tanabe K, Ohnuki M, Narita M, Ichisaka T, et al. (2007) Induction of pluripotent stem cells from adult human fibroblasts by defined factors. Cell 131: 861-872.

9. Yu J, Vodyanik MA, Smuga-Otto K, Antosiewicz-Bourget J, Frane JL, et al. (2007) Induced pluripotent stem cell lines derived from human somatic cells. Science 318: 1917-1920.

10. Dimos JT, Rodolfa KT, Niakan KK, Weisenthal LM, Mitsumoto H, et al. (2008) Induced pluripotent stem cells generated from patients with ALS can be differentiated into motor neurons.Science 321: 1218-1221.
CHY XLL. Contributed reagents/materials/analysis tools: APX YW. Wrote the paper: XLY HS XLL WQL.

11. Karumbayaram S, Novitch BG, Patterson M, Umbach JA, Richter L, et al. (2009) Directed differentiation of human-induced pluripotent stem cells generates active motor neurons. Stem Cells 27: 806-811.

12. Soldner F, Hockemeyer D, Beard C, Gao Q, Bell GW, et al. (2009) Parkinson's disease patient-derived induced pluripotent stem cells free of viral reprogramming factors. Cell 136: 964-977.

13. Hu BY, Weick JP, Yu J, Ma LX, Zhang XQ et al. (2010) Neural differentiation of human induced pluripotent stem cells follows developmental principles but with variable potency. Proc Natl Acad Sci 107: 4335-4340.

14. Onorati M, Camnasio S, Binetti M, Jung CB, Moretti A, et al. (2010) Neuropotent self-renewing neural stem (NS) cells derived from mouse induced pluripotent stem (iPS) cells. Mol Cell Neurosci 43: 287-295.

15. Hanna J, Wernig M, Markoulaki S, Sun CW, Meissner A, et al. (2007) Treatment of sickle cell anemia mouse model with iPS cells generated from autologous skin. Science 318: 1920-1923

16. Wernig M, Zhao JP, Pruszak J, Hedlund E, Fu D, et al. (2008) Neurons derived from reprogrammed fibroblasts functionally integrate into the fetal brain and improve symptoms of rats with Parkinson's disease. Proc Natl Acad Sci 105: 5856-5861.

17. Di Giorgio FP, Carrasco MA, Siao MC, Maniatis T, Eggan K (2007) Non-cell autonomous effect of glia on motor neurons in an embryonic stem cell-based ALS model. Nat Neurosci 10: 608-614.

18. LiW, Qin J, Li X, Zhang L, Liu C, et al. (2010) A versatile tool for tracking the differentiation of human embryonic stem cells. Front Biol 5: 455-463.

19. Wichterle H, Lieberam I, Porter JA, Jessell TM (2002) Directed differentiation of embryonic stem cells into motor neurons. Cell 110: 385-397.

20. Miles GB, Yohn DC, Wichterle H, Jessell TM, Rafuse VF, et al. (2004) Functional properties of motoneurons derived from mouse embryonic stem cells. J Neurosci 24: 7848-7858. 